\section{TCH-009 DEVELOPMENT OF A STABLE NYSTATIN ORAL SUSPENSION TO OVERCOME SHORTAGES OF THE COMMERCIAL MEDICINE}

doi:10.1136/ejhpharm-2013-000276.200

'S Cirillo, ${ }^{1} \mathrm{MM}$ Giacomotti, ${ }^{2} \mathrm{~A}$ Leggieri, ${ }^{3 \mathrm{~F}}$ Bordino, ${ }^{3 \mathrm{D}}$ Chirio, ${ }^{3} \mathrm{M}$ Gallarate. ${ }^{1} \mathrm{ASLTO2}$ NORD, S.C. Farmacia Ospedaliera 1, Turin, Italy; ${ }^{2}$ ASLTO2, Farmacia Ospedaliera Ospedale Maria Vittoria-Ospedale S.G. Bosco, Turin, Italy: ${ }^{3}$ Università degli Studi di Torino, Dipartimento di Scienza e Tecnologia del Farmaco, Turin, Italy

Background Nystatin is often used in the treatment of cutaneous, vaginal, mucosal and oesophageal Candida infections. It's widely employed in cancer and immunocompromised patients suffering from mucositis.

The unobtainability of the commercial oral suspension from July 2011 to February 2012 caused difficulties in the provision of the medicine for these types of inpatients and outpatients.

Purpose With the aim of ensuring a safe continuity of treatment, liquid formulations of nystatin 100,000 IU/ml were developed as oral suspensions, due to the insolubility of the drug in water. The suspensions obtained were studied to assess their chemical-physical stability to find the most suitable formulation.

Materials and Methods Nystatin was dispersed in water containing preservative using carboxymethyl cellulose (CMC) or tragacanth gum as suspending agents. The aqueous vehicles used were sucrose syrup or sorbitol syrup (for the treatment of diabetic or paediatric patients) flavoured with raspberry flavour. The final $\mathrm{pH}$ was maintained in the 7.0-7.8 range. The suspensions obtained were submitted to stability studies determining their chemical-physical properties (mean particle sizes, viscosity, Zeta potential) and the active ingredient content (HPLC analysis) over a 3-month period.

Results Stable suspensions of nystatin were obtained with mean sizes slightly greater than $1 \mu \mathrm{m}$, with both suspending agents and vehicles. CMC and sucrose syrup-containing suspension, however, was more resistant to microbiological attack and it was chosen as the most suitable preparation. Particle sizes, Zeta potential and viscosity remained unchanged for at least 3 months at 25 and $40^{\circ} \mathrm{C}$. The nystatin content in the suspension decreased by about $16 \%$ after the first month and then remained constant over time.

Conclusions The development of a stable nystatin suspension was crucial to ensure continuity of care for patients with oral mucositis previously treated with a commercial formulation, whose temporary lack offered new formulation challenges to the hospital pharmacists.

No conflict of interest.

\section{TCH-010 DEVELOPMENT OF A TOPICAL LIDOCAINE STERILE FORMULATION 20\% (W/V)}

\section{doi:10.1136/ejhpharm-2013-000276.201}

${ }^{1} \mathrm{P}$ Trindade, ${ }^{1} D$ Mateus, ${ }^{1} \mathrm{H}$ Gonçalves, ${ }^{2} A$ Salgado, ${ }^{2} A$ Almeida, ${ }^{3} P$ Machado, ${ }^{2} \mathrm{~J}$ Marto, ${ }^{3} \mathrm{~A}$ Silva, ${ }^{2} \mathrm{C}$ Eleutério, ${ }^{\prime} \mathrm{A}$ Melo Gouveia. ${ }^{1}$ Instituto Português de Lisboa Francisco Gentil EPE, Pharmacy, Lisbon, Portugal; ${ }^{2}$ iMed.UL - Research Institute for Medicines and Pharmaceutical Sciences, Nanomedicine and Drug Delivery Systems, Lisbon, Portugal; ${ }^{3}$ Faculty of Pharmacy University of Lisbon, Microbiology, Lisbon, Portugal

Background The topical use of concentrated solutions of lidocaine (4 to 20\%), which are usually unavailable, has been reported for microvascular surgery. Vasospasm is known to have an adverse effect on the survival of free tissue transfers. Prolonged vasoconstriction decreases blood flow to the flap and promotes thrombosis at the anastomotic site. The wide availability and rapid effect of topically applied lidocaine is used by many surgeons to prevent and correct vasospasm.

Purpose To compound a sterile $20 \%$ (w/v) lidocaine solution physicochemically and microbiologically stable for topical application during surgery.
Materials and Methods Three batches of a 20\% (w/v) sterile lidocaine solution were prepared using two sterilisation steps: autoclaving followed by filtration $(0.22 \mu \mathrm{m})$ inside a horizontal laminar flow hood. Packaging in $10 \mathrm{ml}$ dropping containers prevents intravenous administration and ensures a maximum safe dose $(2 \mathrm{~g})$. For physicochemical and microbiological stability studies, samples were stored in the dark at $5 \pm 3^{\circ} \mathrm{C}$ and $22 \pm 3^{\circ} \mathrm{C}$, for 15 days. Sterility tests and bacterial endotoxins assays were performed (Ph. Eur.). Samples were collected and characterised on days 0 (T0), 7 (T7) and 15 (T15). Colour, odour, appearance, $\mathrm{pH}$, osmolarity, density and lidocaine hydrochloride content were analysed.

Results Throughout the study, the $20 \%$ (w/v) lidocaine hydrochloride solutions remained clear, colourless, limpid and odourless The $\mathrm{pH}$ of the solutions stored at $5 \pm 3^{\circ} \mathrm{C}$ was $3.6 \pm 0.04$ (T0), $3.8 \pm 0.08$ (T7), $3.9 \pm 0.02$ (T15), and $3.6 \pm 0.04$ (T0), $3.9 \pm 0.02$ (T7), $4.0 \pm 0.03$ (T15) for the solutions maintained at $22 \pm 3^{\circ} \mathrm{C}$ The HPLC analyses showed that the lidocaine hydrochloride content was maintained (90-110\%) after 15 days in all conditions tested. Density and osmolality remained constant, i.e. $1.0049 \pm 0.0036 \mathrm{~g} / \mathrm{cm}^{3}$ and $1175.3 \pm 20.2 \mathrm{mOsm} / \mathrm{kg}$, respectively $(\mathrm{n}=3)$. The three batches proved to be sterile and endotoxins-free during the study.

Conclusions The lidocaine hydrochloride solution proved to be physicochemically and microbiologically stable for 15 days stored in the dark.

No conflict of interest.

\section{TCH-011 DEVELOPMENT OF PYRIDOXAL-5-PHOSPHATE HARD CAPSULES FOR PAEDIATRIC USE}

doi:10.1136/ejhpharm-2013-000276.202

C Serrano, P Joret, V Siorat, P Vaconsin, T Abarou, T Storme. Hopital Robert Debre (Ap-Hp), Pharmacy, Paris, France

Background The active form of vitamin B6, called pyridoxal5 -phosphate (P5P), is an essential cofactor for several enzymes involved in various pathways of intermediary metabolism. PNPO is the rate-limiting enzyme in the synthesis of pyridoxal from vitamin B6 and a lack of activity causes dependency on an external source of pyridoxal. Epileptic seizure is the clinical outcome of $\mathrm{P} 5 \mathrm{P}$ deficiency.

Purpose To provide a dosage form suitable for newborns and children. Capsules containing standardised P5P were compounded. Moreover, a fully soluble powder blend was formulated to fill the capsules and a method to determine the stability of the P5P content was developed.

Materials and Methods Dissolution assays were performed using oral syringes as nurses do. Time to complete dissolution and concentration were determined at each test. P5P content was determined by HPLC-UV $(205 \mathrm{~nm})$. The mobile phase consisted of phosphate buffer $(0.05 \mathrm{M}$; pH 2.6) at a flow rate of $1 \mathrm{ml} / \mathrm{min}$. The right active ingredient was tested by adding vitamin $\mathrm{B} 6$ to samples. Degradation by-products in stress conditions were also determined. The method was validated according to ICH recommendations.

Results Strengths were standardised at 10, 25, 50, 100 or $250 \mathrm{mg} /$ capsule. The adopted blend is quickly solubilised in water and has a sweet taste. The HPLC readings were linear $\left(r^{2}=0.9994\right)$ at the wavelength used, indicating good reproducibility and repeatability $(\mathrm{SD}=0.46 \%)$. No matrix effect due to the diluent was observed.

Conclusions As P5P is a low toxicity compound, a test treatment with P5P is given to every newborn with idiopathic seizure before any treatment with standard antiepileptics. This method allows rapid routine assay of P5P. Stability testing of 3 compounded batches is ongoing.

No conflict of interest. 